\title{
Understanding the acoustic response of deepwater completions
}

AndREY BAKULIN, WesternGeco

Florian Karpfinger and Boris Gurevich, Curtin University of Technology
$\mathrm{D}$ eepwater production often hinges on the ability to safely complete and effectively draw down a small number of very challenging wells. Chances of success are greatly increased if surveillance tools are available to quickly diagnose downhole conditions and detect potential issues early on. Real-time completion monitoring with acoustic waves (RTCM) has great potential for diagnosing problems in sand-screened deepwater completions. RTCM uses tube waves to detect permeability changes and passive noises to characterize perforation flow. Interaction of a single tube wave with permeable formations in open boreholes is well explained by Biot's theory of poroelasticity. However, experimental studies in laboratory models of sand-screened completions reveal that fast- and slow-tube waves behave differently. Further progress in acoustic surveillance requires better understanding on how signatures of fast- and slow-tube waves depend on completion properties. To this end, we simulate the dispersion and attenuation of the two tube waves by examining the solutions of Biot's equations of poroelasticity in cylindrical structures using a spectral method.

Basic concept of evaluating permeability with acoustic waves

At low frequencies, wave propagation in an open borehole is dominated by tube (Stoneley) waves. Waves compress the fluid column and lead to a piston-like motion. When fluid is compressed, it expands radially and pushes against the formation or casing. In the presence of a permeable borehole wall, fluid movement across the interface leads to a slowdown in velocity and an increase in attenuation (Figure 1). These effects are well explained by Biot's theory and form the foundation for estimating near-wellbore permeability from an open-hole acoustic logging.

Sand-screened deepwater completion has a more complicated structure (Figure 2) due to multiple permeable layers. The sand screen represents an additional pipe with a very high permeability. The annulus between the casing and screen is filled with gravel sand or fluid, both of which possess negligible shear modulus. The sand screen and gravel pack prevent migration of reservoir sand into the wellbore and maintain the structure of the reservoir around the wellbore. The presence of two fluid-like columns in the completion gives birth to two tube waves: fast and slow. They have been observed in experiments with full-scale laboratory models of deepwater completion. The most basic task of RTCM is to monitor changes in the permeability of the sand screen and gravel-pack layer. Here we aim to examine the effect of sand-screen permeability on velocities and attenuation of two tube waves.

Experimental observations in models of sand-screened deepwater completions

Bakulin et al. (2008) described experiments in a full-scale

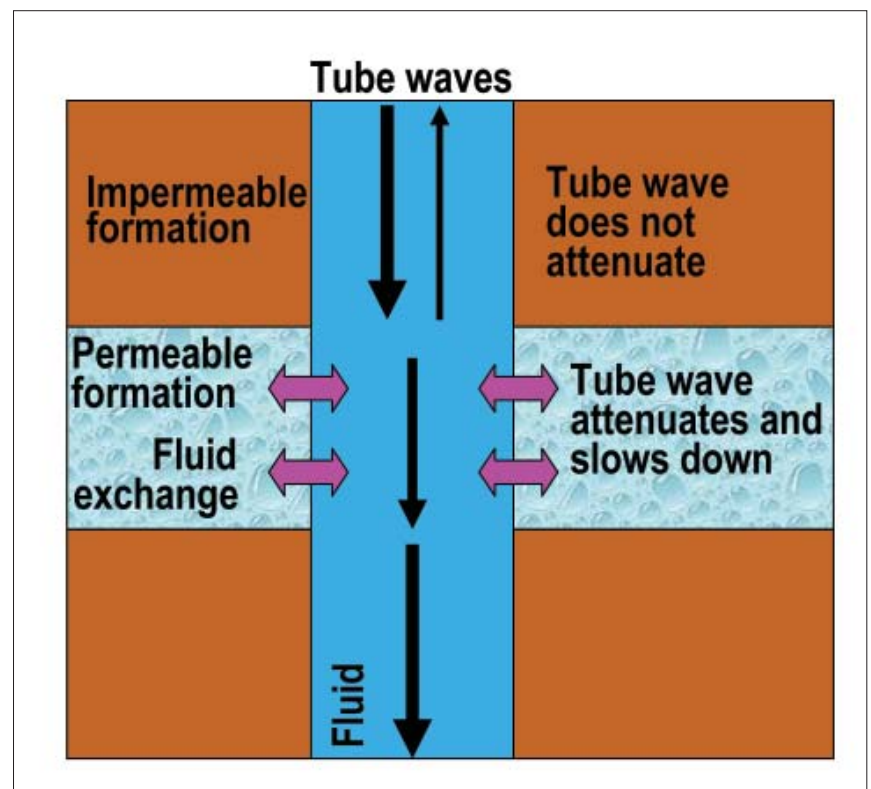

Figure 1. Tube waves increase in attenuation and decrease in velocity when encountering a permeable interval where fluid exchange can occur between borehole and formation.

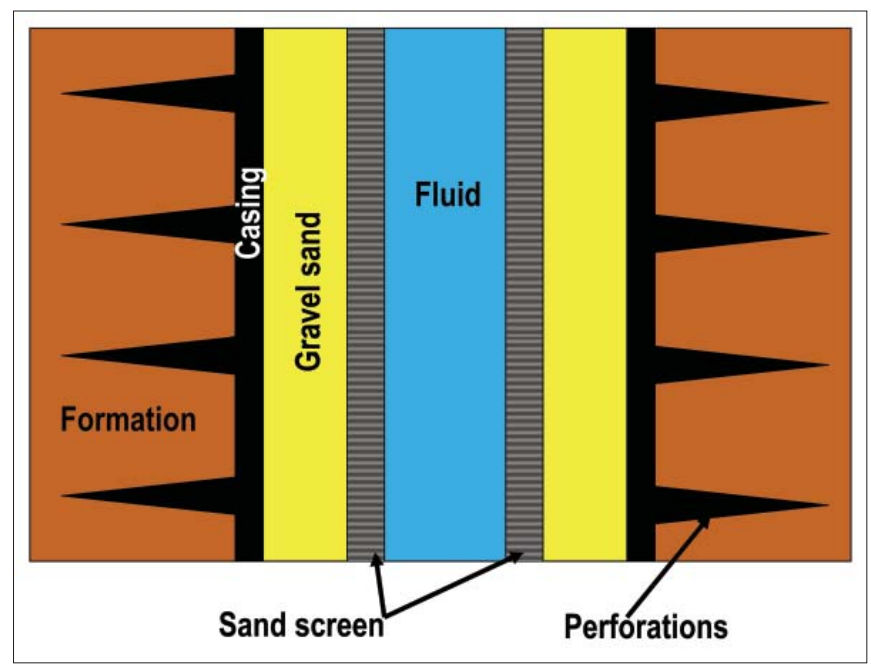

Figure 2. Schematic representation of a cased deepwater well with sand-screened completion.

laboratory model of idealized completion. Figure 3 shows schematics as well as actual photographs of the setup. Table 1 shows the geometry of the model. The outer pipe simulates the casing string, whereas the inner pipe represents the sand screen. The sand screen has aluminum base pipe and plastic wire wraps (Figure 3c). To simulate the impermeable or plugged sand screen, a blank pipe was used (Figure 3d). The casing was impermeable (closed perforations), and the experimental study focused on the effects of the screen permeability on the signatures of the fast- and slow-tube waves. 


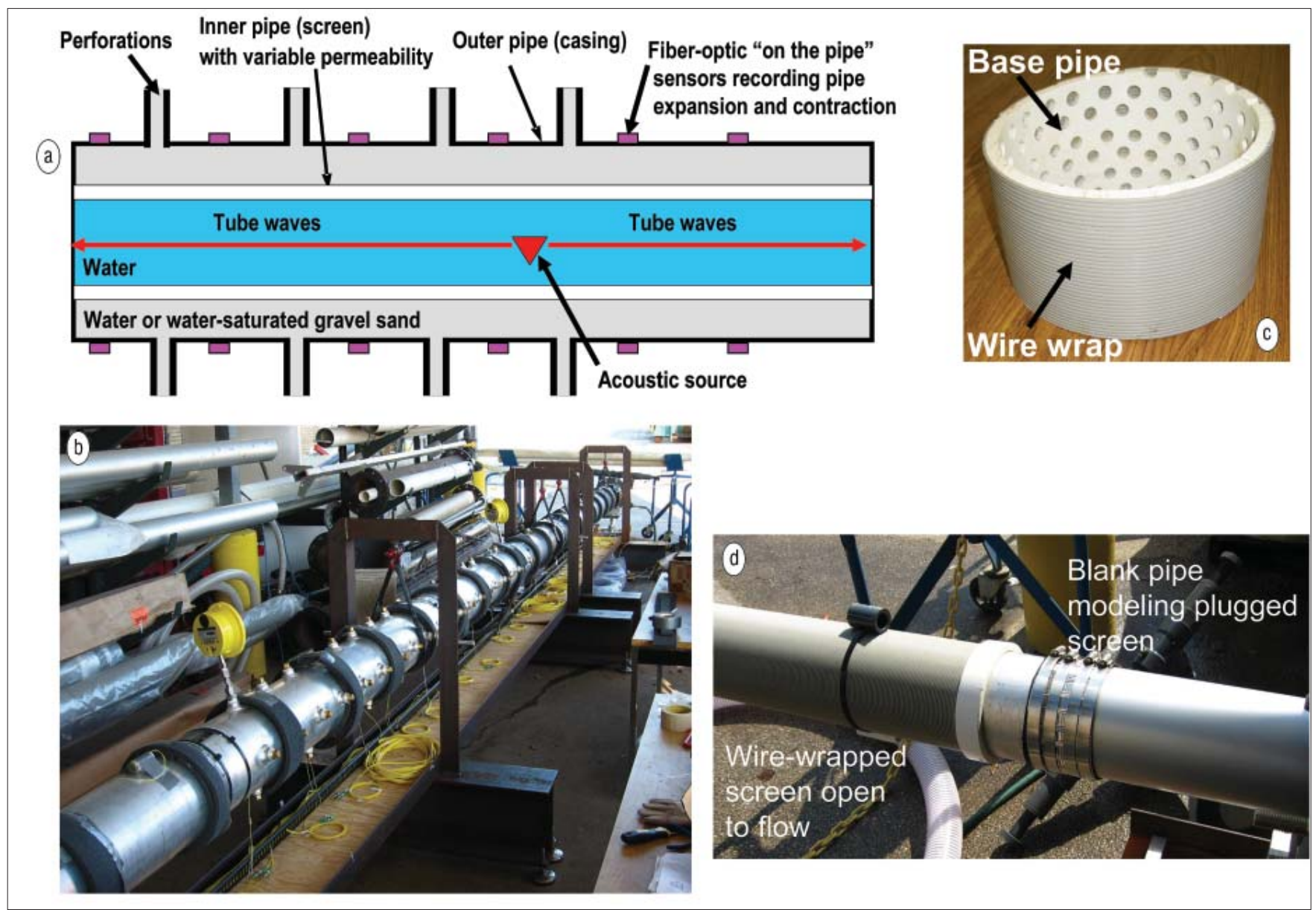

Figure 3. Sketch (a) and photograph (b) of the full-scale laboratory setup used to simulate completed horizontal well; (c) cross-section of the screen showing wire wrap and base pipe (although plastic base pipe is shown, aluminum was used in the experiment); (d) wire-wrapped sand screen open to flow and blank pipe simulating plugged screen.

If the sand screen is impermeable (blank pipe), then theory predicts four wave modes at low frequencies, all without attenuation (Figure 4): two tube waves and two plate (extensional) waves. Two tube waves exist because the completion contains two fluid columns: one inside the screen and the other in the annulus. The two plate waves are supported by the screen (inner pipe) and casing (outer pipe), respectively. If formation is

\begin{tabular}{|l|l|l|}
\hline Cylindrical layers & Material & Outer radius (m) \\
\hline Layer 1 & Water & 0.0635 \\
\hline Layer 2 & Aluminum sand screen & 0.0667 \\
\hline Layer 3 & Water & 0.1032 \\
\hline Layer 4 & Aluminum casing & 0.1095 \\
\hline
\end{tabular}

Table 1. Geometry and material composition of the completion model. added on the outside of the casing, then the casingsupported plate wave disappears, while all three other modes remain.

Experimental seismograms (Figure 5a) confirm the presence of fast- and slow-tube waves. The plate waves have smaller amplitudes and are visible only at higher amplifications. Since the plate waves are not sensitive to permeability, we concentrate on the analysis of the tube-wave signatures.

Figure $5 \mathrm{~b}$ suggests that an open-to-flow sand screen leads to increased attenuation of both fast- and slow-tube waves. This attenuation is further increased when water in the annulus is replaced by gravel sand. Wave propagation in a completion model with impermeable screen is well understood and can be accomplished by a root-finding technique. However, to capture the effects of sand-screen permeability on wave propagation, we need to use more complex constitutive equations that pose substantial challenges for modeling borehole

modes. Following Bakulin et al., we assume that, to the first approximation, sand screen can be modeled as a poroelastic Biot's layer and examine tube-wave signatures as a function of screen permeability. Such signatures are usually derived using an analytical root-finding technique (Paillet and White, 1982) that is difficult to implement for multilayered attenuative poroelastic structures. The spectral method is a new approach to bypass the difficulties of root-finding for fluid and elastic cylindrical structures and was recently extended to poroelastic cylindrical structures (Karpfinger et al., 2008). By directly discretizing the underlying differential equations in the radial direction, the spectral method handles such complicated cylindrical structures very effectively and gives phase velocities and attenuation of all modes as a function of frequency. 
Effect of screen permeability on tube waves

Let us examine how velocities and attenuation of two tube waves depend on the screen permeability, and compare them with the behavior of these signatures for a single tube wave in the case of an open borehole surrounded by infinite fluidsaturated and permeable formation (Winkler et al., 1989). In the open-hole model at low frequencies, tube-wave velocity decreases and attenuation increases with increasing forma-

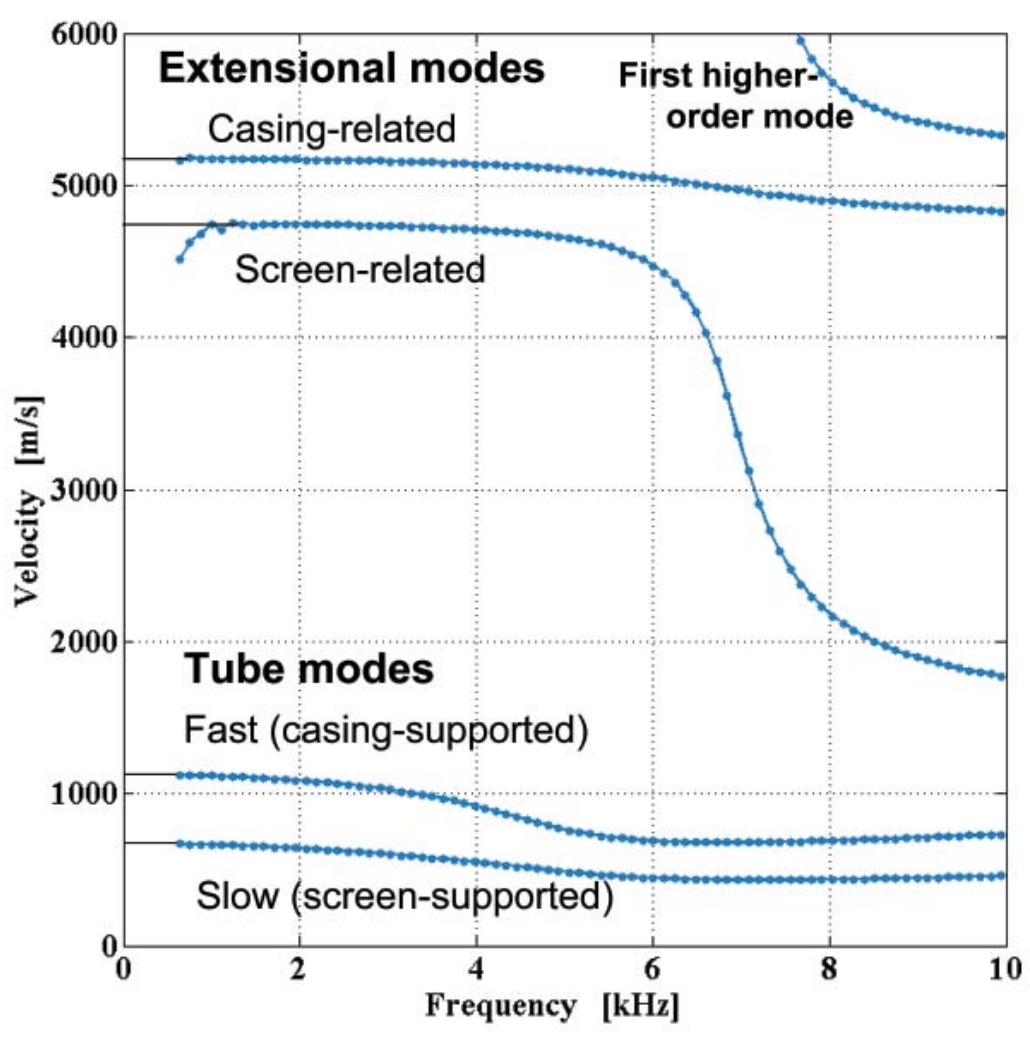

Figure 4. Dispersion curves for an idealized completion model (water-impermeable screenwater-casing-vacuum) when sand screen has vanishing permeability. Note that all modes are completely lossless. Two slowest modes represent tube waves associated with the two fluid columns whereas two fastest modes represent plate (extensional) modes in screen and casing tubulars, respectively. tion permeability. In a completion model, we have two tube waves, and their behavior is different.

Figures 6 and 7 show modeled velocity and attenuation of fast- and slow-tube waves as a function of screen permeability. Let us first examine the fast-tube wave, which is mainly supported by the casing. In the low-permeability limit, the screen behaves as an impermeable pipe, and the fast-tube wave experiences no loss as already shown in Figure 4. At the other extreme, when the screen is very permeable, it provides almost no resistance to the radial fluid motion across the screen and behaves like a layer of liquid. Therefore we expect to have a single (fast) tube wave supported by casing, again without a loss. From this transition, we deduce that the fast-tube wave is mainly supported by the casing. What would happen for intermediate permeabilities?

For subdarcy permeabilities, attenuation of the fast-tube wave increases with increasing permeability. However, this elevated attenuation peaks at about 1.5 darcy and then decreases, returning to the state of virtually no attenuation at large permeability (Figure 6b). To a first order, the location of the attenuation maximum is controlled by screen permeability and thickness. Radial profiles of displacement provide an additional insight on a possible mechanism for this attenuation at intermediate permeabilities. For low permeabilities, axial displacements of the fast wave are of the same sign, but of different magnitudes (Figure 8a). When the screen becomes more permeable, the different rates of compression inside the two liquid columns lead to a fluid exchange across the screen. This exchange particularly intensifies for permeabilities

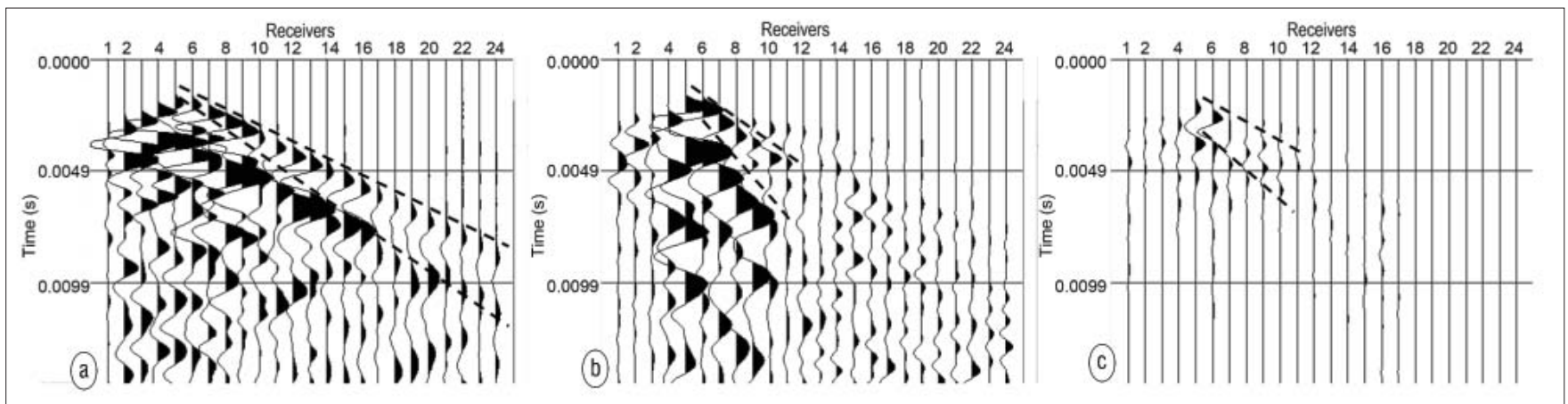

Figure 5. Experimental seismograms in different completion models from the same source near receiver 5 shown with the identical magnification: (a) entire completion has impermeable screen (blank pipe) with no gravel sand; (b) blank pipe between receivers 1 and 12 is replaced by a section of open-to-flow wire-wrapped screen; (c) same as (b) but with gravel pack. Note that two arrivals of fast-and slow-tube waves are visible on both displays. For an impermeable screen without sand (a) velocities are $-1000 \mathrm{~m} / \mathrm{s}$ and $700 \mathrm{~m} / \mathrm{s}$, but for an open screen with and without sand $(b-c)$ they are slower $(-700-800 \mathrm{~m} / \mathrm{s}$ and $400-600 \mathrm{~m} / \mathrm{s}$, respectively). Observe increase in attenuation with introduction of screen section (b) and even larger increase when adding sand (c). 

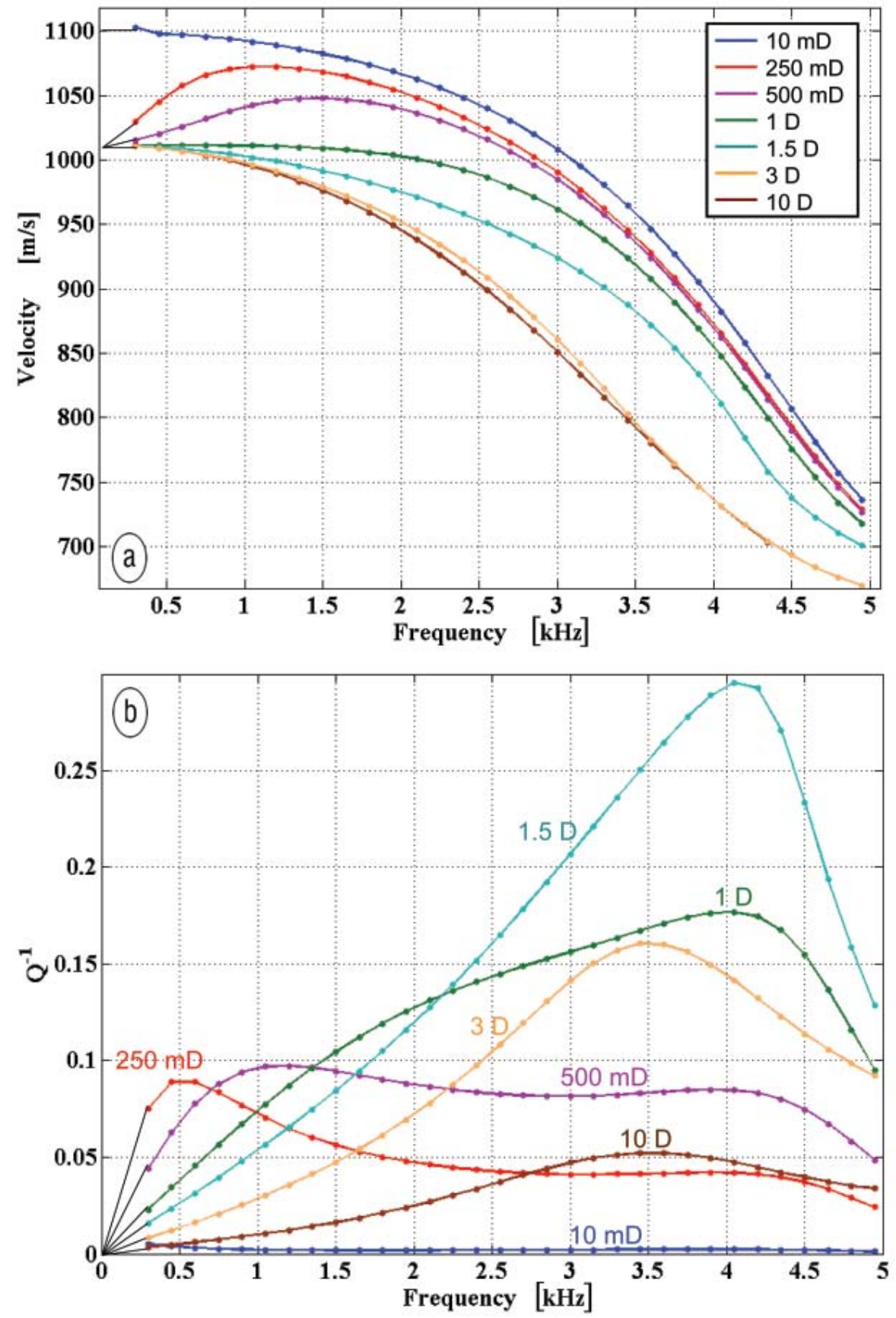

Figure 6. Velocity (a) and attenuation (b) of the fast-tube wave mode as a function of screen permeability in an idealized completion model (water-permeable screen-water-casing-vacuum). Dots denote computations by spectral method. Thin black lines show extrapolated behavior at low frequencies where current implementation of the spectral method is numerically unstable.

$(\sim 1.5$ darcy $)$ where the attenuation reaches maximum and is also manifested by rapid equalization of axial displacements occurring in this region. At higher permeabilities, the axial displacement levels off, while the radial displacement resembles the linear profile typical for a conventional tube wave in a fluid-filled cylinder with weak dispersion and no attenuation (Figure 8b).

As for the fast-tube wave velocity, it slows down progressively, with most changes occurring between 250 mdarcy and 3 darcy (Figure 6a). While one normally expects velocity slowdown with the increased attenuation, it is unusual to observe that velocity continues to decrease even when attenuation starts to drop between 1.5 and 10 darcy.

The slow-tube wave demonstrates a behavior that is more similar to that of a single tube wave in an open borehole surrounded by a permeable formation. Attenuation is increasing progressively with increasing permeability (Figure 7b). While in open borehole, the tube wave always remains a propagating mode $\left(\mathrm{Q}^{-1} \leq 2\right.$, e.g., wave attenuates within more than one wavelength), in a completion the slow-tube wave may become nonpropagatory $\left(\mathrm{Q}^{-1}\right.$ $>2$ ) at a finite frequency and thus attenuates within less than a wavelength (Figure 7b). Such behavior of the slow mode can be readily explained by radial profiles of displacement (Figure 8). One can observe a pistonlike motion with large axial displacement and small radial one. However, those pistons move in opposite directions in the inner and outer fluid columns, which are manifested by the opposite signs of the axial displacements in Figure 8. This distinct feature makes slow-tube mode analogous to a Biot's slow body wave. When the screen becomes permeable, this out-of-phase motion in the two columns naturally leads to an elevated fluid communication between two liquid columns. As a result, we observe rapid increase in attenuation (Figure $7 \mathrm{~b}$ ) and eventually a complete absorption of the slow mode at higher permeabilities. Despite amplitude decay of the slow wave, the character of the profile, and in particular, the opposite signs of the displacements remain stable at all permeabilities (compare Figure 8a and $8 \mathrm{~b}$ ).

In line with the increased attenuation at low frequencies, the velocity of the slow-tube wave drastically decreases (Figure 7a). One important observation is that at zero frequency, the slow-tube wave velocity approaches a positive value, where- 


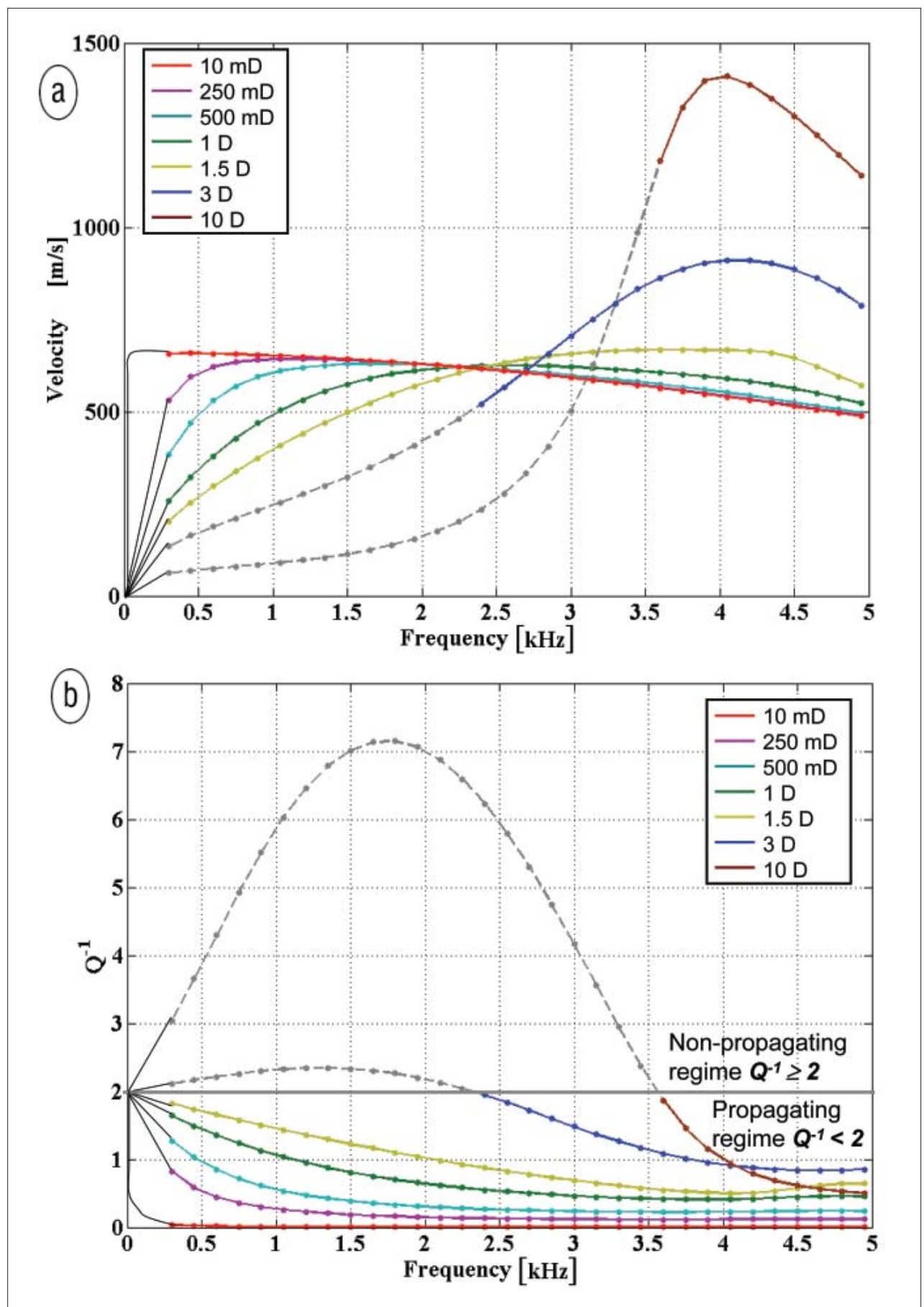

Figure 7. Velocity (a) and attenuation (b) of slow-tube wave mode as a function of screen permeability in an idealized completion model (water-permeable screen-water-casing-vacuum). Notation is the same as in Figure 6. Horizontal gray line $\left(Q^{-1}=2\right)$ separates nonpropagating $\left(Q^{-1} \geq 2\right)$ and propagating regimes $\left(Q^{-1}<2\right)$. In addition, curves are grayed out for frequencies where the slow mode becomes nonpropagating and attenuates within less than a wavelength.

as for a finite permeability, it is expected to vanish at zero frequency similarly to the tube wave in an open borehole. Current implementation of the spectral method (Karpfinger, 2008) becomes numerically unstable at very low frequencies, and therefore these conclusions are tentative and need to be verified by additional research. Interestingly, at higher frequencies, the velocity of the slow mode increases with permeability increase (Figure 7a) and may even exceed the imper- meable limit. This has been also observed for a simpler model of open borehole in fluid-saturated formation (Winkler and Johnson, 1989).

\section{Discussion and conclusions}

Modeling with spectral method confirms strong dependence of fast- and slow-tube wave signatures on the screen permeability. Therefore, an acoustic surveillance system installed in a deepwater completion has a great chance to detect sandscreen plugging in real time. Sand-screen plugging is a serious problem that can create high skin factor for a well and cause a substantial decrease in production rates and thus well underperformance. However, without surveillance, it is virtually impossible to identify the source of the well underperformance, since it may be caused by other completion problems such as perforation plugging, near-wellbore permeability reduction (formation damage), or large-scale issues such as reservoir compartmentalization. Real-time completion monitoring with acoustic waves may reveal, actual source of well underperfomance and thus lead to a safer and more prolific drawdown and production strategies. RTCM has the potential to revolutionize our ability to manage deepwater wells by understanding evolution of flow, drawdown, and impairment in real time.

To accomplish these ambitious goals, we need to have good understanding of how acoustic signatures of interest depend on permeability of various completion layers. In this study, we concentrated on the analysis of the simplest completion model without gravel pack, and assumed that the sand screen can be modeled as a poroelastic Biot's layer. We verified that in this case the wave propagation is dominated by fast- and slow-tube modes supported by casing and screen, respectively. We studied the effect of screen permeability on the velocity and attenuation of fast- and slow-tube waves. For the slow-tube wave, veloc- 

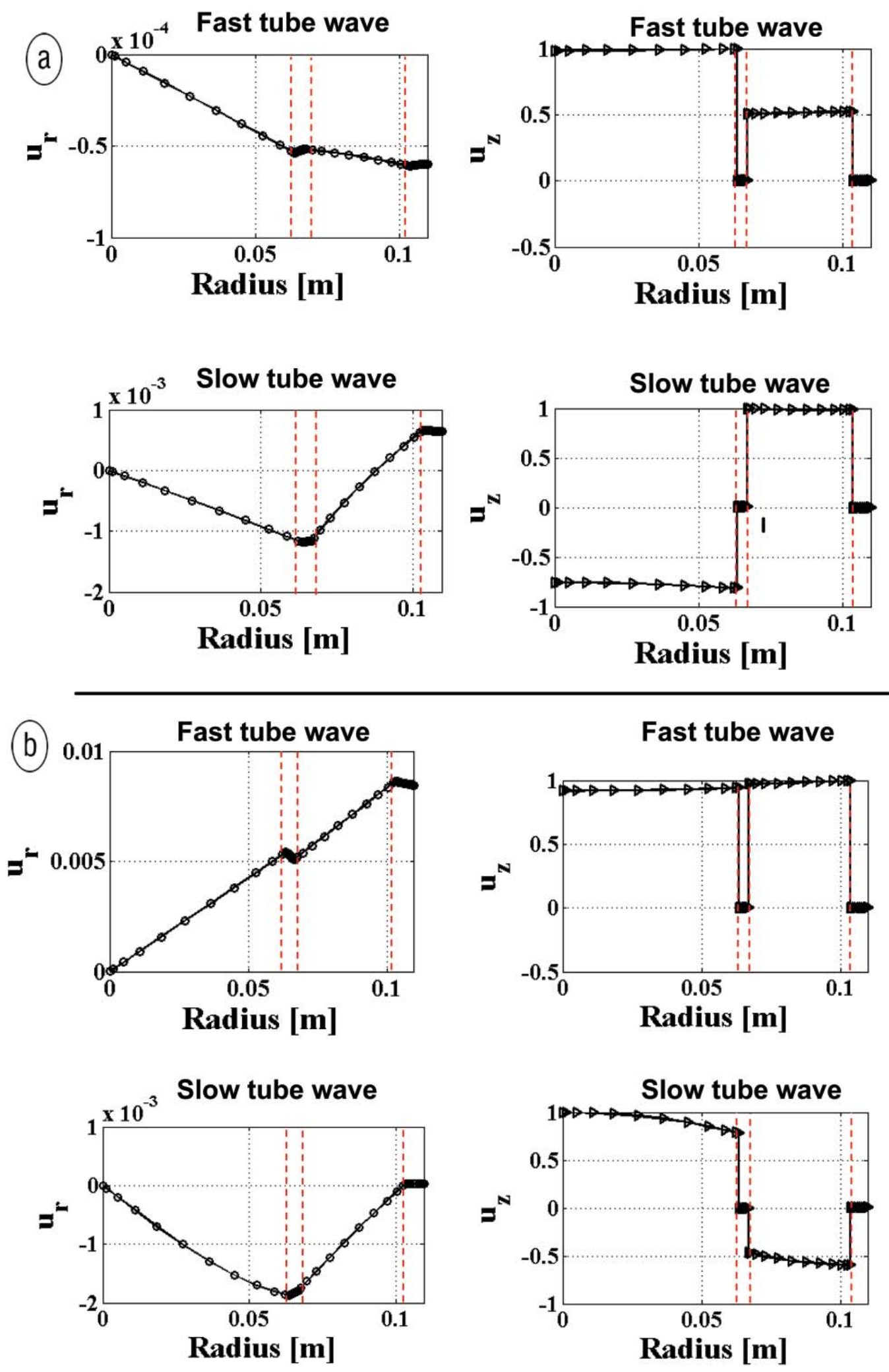

Figure 8. Radial variation of displacements at $1000 \mathrm{~Hz}$ in fast-and slow-tube wave modes for a model of sand-screened completion without gravel pack with different screen permeabilities: (top) 10 mdarcy, (bottom) 5 darcy. For a poroelastic screen, the displacement averaged over solid and fluid phase is shown that is continuous at the open interface with a fluid. Note that increasing permeability equalizes axial displacement of the fast wave in both fluid columns, and leads to a linear profile of radial displacement. In contrast, axial displacement in the slow wave maintains opposite signs in two fluid columns, which creates fluid communication and eventually leads to a complete dissipation of this mode at higher permeabilities. 
ity decreases and attenuation increases with increasing screen permeability. Such behavior is caused by escalating fluid communication between two fluid columns and can be explained by opposite signs of axial fluid displacements on different sides of the screen. In contrast, the fast-tube wave experiences moderate attenuation at some characteristic frequency, which is controlled by screen permeability and thickness, whereas for small and large permeability fast-tube wave remains free of losses. In the limit of high permeability, the fast-tube wave transforms into a regular tube wave as if the sand screen becomes part of the surrounding liquids.

These modeling results are in a qualitative agreement with experimental observations in full-scale laboratory models of deepwater completions. The main discrepancy is related to the behavior of the slow-tube wave. Simple calculation of static screen permeability suggests values larger than 100 darcy for which modeling predicts complete dissipation of the slow mode (Figure 7b). However experiments reveal that slow-tube waves are observed and survive even when water in the annulus is replaced by water-saturated gravel sand. Better model of sand screens is required to reconcile this discrepancy. Understanding the connection between static and "dynamic" or "acoustic" permeability for meso-scale structures such as sand screens and perforated casing is a key to such reconciliation.

Suggested reading. "Real-time completion monitoring with acoustic waves" by Bakulin et al. (Geophysics, 2008). "Acous- tic waves in sand-screened deepwater completions: Comparison of experiments and modeling" by Bakulin et al. (Geophysics, 2008). "Downhole acoustic surveillance of deepwater wells" by Bakulin et al. (TLE, 2008). "Modeling of wave dispersion along cylindrical structures using the spectral method" by Karpfinger et al. (Journal of Acoustical Society of America, 2008). "Modeling of axisymmetric wave modes in a poroelastic cylinder using spectral method" by Karpfinger et al. (Journal of the Acoustical Society of America Express Letters, 2008). "Acoustic modes of propagation in the borehole and their relationship to rock properties" by Paillet and White (Geophysics, 1982). "Permeability and borehole Stoneley waves: Comparison between experiment and theory" by Winkler et al. (Geophysics, 1989). TEE

Acknowledgments: We are grateful to Boris Kashtan (St. Petersburg State University, Russia) who suggested the idea of applying the spectral method to the problem at hand and Richard Craster (Imperial College, London) for helpful advice. We greatly appreciate discussion on the physics of tube waves with David L. Johnson (Schlumberger-Doll Research). Florian Karpfinger thanks Shell International Exploration and Production for support of his PhD project. Andrey Bakulin was with Shell International E\&P when this work was performed.

Corresponding author: florian.karpfinger@postgrad.curtin.edu.au 